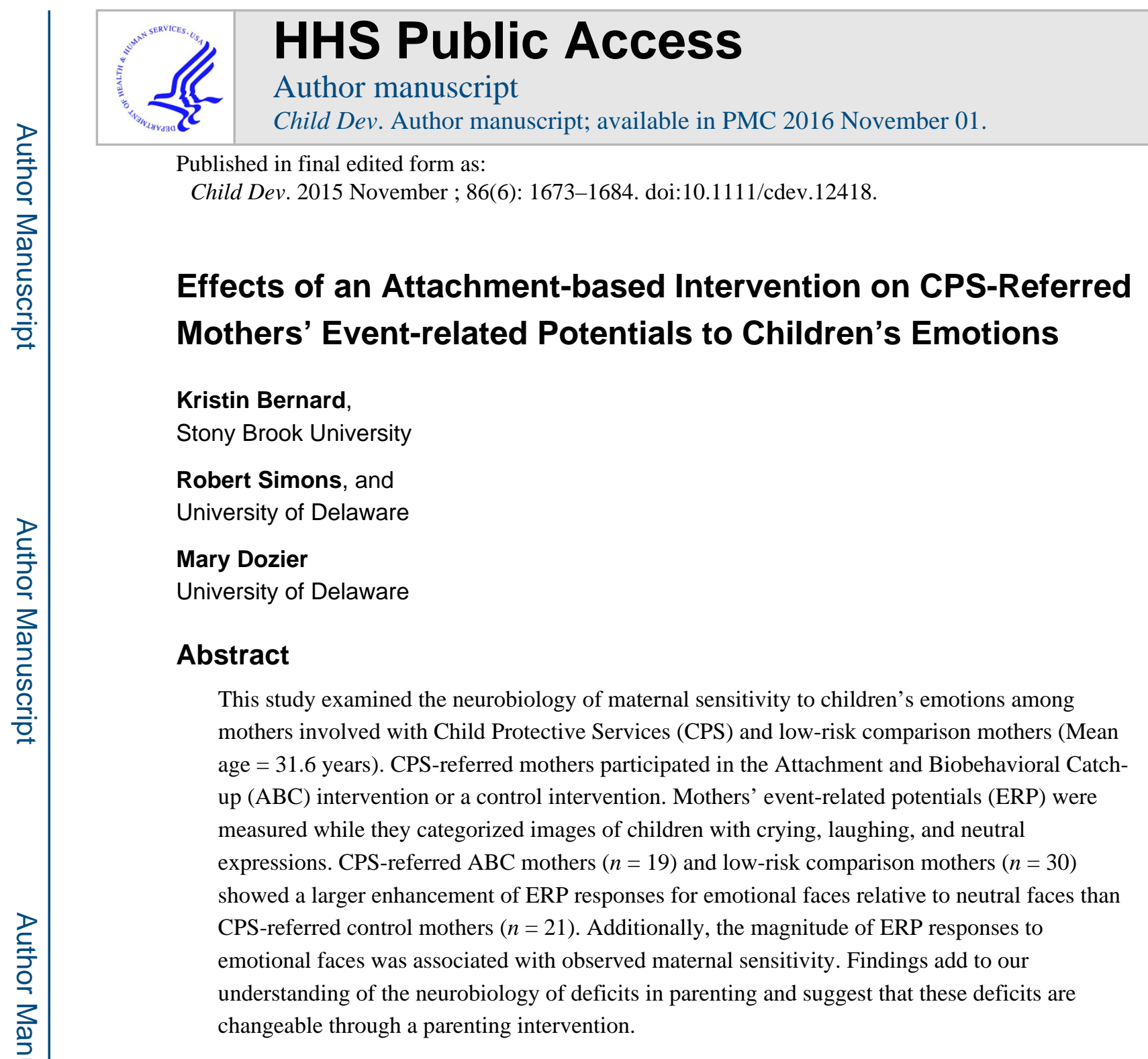

Neglecting mothers often struggle to respond sensitively and synchronously to their children's cues. Although these deficits have been most often measured through behavioral observation, there is increasing attention being directed toward understanding the neurobiology of optimal and atypical maternal care (Barrett \& Fleming, 2011; Swain, Lorberbaum, Kose, \& Strathearn, 2007). The Attachment and Biobehavioral Catch-up intervention (ABC; Dozier \& the Infant Caregiver Project Lab, 2006) for mothers identified as at risk for neglecting their infants aims to enhance mothers' sensitivity to children's distress and increase mothers' synchronous responding to children's signals. For the present study, we used event-related potential (ERP) methodology to examine the degree to which the $\mathrm{ABC}$ intervention enhanced mothers' neurobiological processing of children's emotional signals.

Corresponding author: Kristin Bernard, Department of Psychology, Stony Brook University, Stony Brook, NY 11794, kristin.bernard@stonybrook.edu.

The content is solely the responsibility of the authors and does not necessarily represent the official views of the National Institute of Mental Health or the National Institutes of Health. 


\section{Neurobiology of Maternal Behavior}

Beyond the obvious failure in caregiving related to neglect itself, mothers identified as maltreating (i.e., neglecting and/or abusive) also show deficits in responding sensitively to their children's signals. More specifically, mothers at risk for maltreating their children tend to show lower levels of maternal sensitivity to children's distress than non-maltreating mothers (Cicchetti, Rogosch, \& Toth, 2006; Lyons-Ruth, Connell, Zoll, \& Stahl, 1987). They may respond with hostile or contradictory emotional responses, or fail to respond much at all (Edwards, Shipman, \& Brown, 2005). Maltreating mothers also tend to express less delight in their children than non-maltreating mothers, as evidenced by flat affect, lack of verbal and physical affection, and poor contingent responsiveness to children's signals (Gaudin, Polansky, Kilaptrick, \& Shilton, 1996; Milot, St-Laurent, Ethier, \& Provost, 2010). These behavioral deficits in maternal sensitivity may contribute to elevated rates of insecure and disorganized attachments among maltreated children (De Wolff \& van IJzendoorn, 1997; Hesse \& Main, 2006).

A number of fMRI studies have examined the neurobiology of maternal sensitivity by looking at mothers' brain activity in response to a range of stimuli, such as pictures of children in distress or auditory cues of children's cries (e.g., Lorberbaum et al., 2002; Noriuchi, Kikuchi, \& Senoo, 2008). Mothers show activation across brain regions that are implicated in emotion perception, response initiation, and stimulus valence, including the anterior cingulate, medial and orbitofrontal cortex, amygdala, thalamus, midbrain, and striatum (Swain et al., 2007).

In an ERP study, Proverbio, Brignone, Matarazzo, Del Zotto and Zanil (2006) found that the amplitude of mothers' N170 (negative deflection occurring approximately 130-200 ms after the stimulus) was significantly larger for images of children showing strongly negative emotions (i.e., distress) relative to less negative emotions (i.e., discomfort). Of particular relevance to the current study, Rodrigo et al. (2011) compared N170 responses of 14 neglectful and 14 control mothers as they categorized pictures of infant expressions (i.e., crying, laughing, neutral). Whereas control mothers had higher amplitude (more negative) N170 to crying faces than to neutral and laughing faces, the N170 in neglectful mothers did not vary with the emotional expression. Thus, neglectful mothers did not show differentiated processing of infants' emotions at this early perceptual stage, but rather they responded similarly across emotional expressions.

Later ERP components that reflect evaluation of stimuli or more sustained attention allocation have also been implicated in mothers' processing of children's signals of distress. Proverbio et al. (2006) found that mothers' P300 responses (positive deflection extending from approximately $300 \mathrm{~ms}$ to $600-700 \mathrm{~ms}$ after the stimulus) were significantly larger for strongly negative emotions relative to positive (i.e., pleasure, comfort) and weakly negative (i.e., discomfort) expressions. This component might be better labeled as the late positive potential (LPP) because it reflects a sustained positivity to emotional information and persists beyond the window of the traditional P300 elicited by oddball paradigms (Hajcak, MacNamara, \& Olvet, 2010). Given evidence of mothers' enhanced N170 and LPP responses to negative emotional expressions, these components may be informative in 
understanding the physiology of parenting -- specifically, maternal sensitivity to children's emotional displays.

\section{Intervening to Enhance Maternal Sensitivity and Delight and Synchrony}

The $\mathrm{ABC}$ intervention is a 10-session intervention delivered in families' homes that aims to enhance maternal sensitivity among CPS-referred mothers. Maternal sensitivity was targeted in order to support children's development of secure organized attachments as well as behavioral and biological regulation. In a randomized clinical trial, children in the $\mathrm{ABC}$ intervention showed higher rates of organized, secure attachments, and more normative cortisol regulation, than children who received a control intervention (Bernard et al, 2012; Bernard, Dozier, Bick, \& Gordon, in press).

\section{The Present Study}

The present study examined mothers' ERP responses (N170 and LPP) while they viewed pictures of children with different emotional expressions. We hypothesized that low-risk mothers would show larger ERPs to emotional faces than neutral faces, whereas CPSreferred mothers would fail to show this discrimination between conditions. We expected that CPS-referred mothers who received the ABC intervention would show larger ERPs to emotional faces than neutral faces, similar to low-risk mothers. Finally, across groups, we expected that behavioral ratings of maternal sensitivity would be associated with mothers' ERP responses, with more sensitive mothers showing larger ERPs to emotional faces than neutral faces, relative to less sensitive mothers.

\section{Method}

Participants

The full sample of participants enrolled in this study included 86 mothers: 52 mothers involved with CPS following allegations of neglect primarily living in a large mid-Atlantic city and 34 low-risk mothers with no history of CPS involvement recruited primarily from suburban communities. The 52 mothers from the CPS-referred group were participating in an ongoing longitudinal study assessing the efficacy of the $\mathrm{ABC}$ intervention. We did not have access to case records, so were unable to further characterize the sample regarding maltreatment history, substantiation, and other risk factors. Given research suggesting that likelihood of re-reporting to CPS and negative outcomes look similar among children regardless of whether CPS reports are substantiated (e.g., Hussey et al., 2005), we considered all CPS-referred children to be in need of intervention. Approximately half of the CPS-referred mothers received the $\mathrm{ABC}$ intervention $(n=25)$ and half received the control intervention, Developmental Education for Families (DEF; $n=27$ ). As described below, the final sample for primary analyses included 70 participants (ABC $n=19$, DEF $n=21$, Lowrisk $n=30$ ), due to some participants being excluded because of non-useable ERP data. Descriptive statistics for demographic data (e.g., race/ethnicity, education level, income, age) for each group are presented in Table 1. 
Participant recruitment-The present study included a sub-sample of CPS-referred parents selected from the ongoing randomized clinical trial. Enrollment in the larger randomized clinical trial began in January 2006. Data collection for the present study (i.e., home visits for behavioral measures and lab visits for ERP assessments) was completed between November 2011 and June 2012. Parents with children between 4 and 6 years old at the time of this study were invited to participate in the order that they completed the intervention (with parents most recently completing the intervention contacted first). The same procedure for identifying and contacting parents was followed for both intervention groups. As is reported in previous reports (e.g., Lind, Bernard, Ross, \& Dozier, 2014), session completion and retention for follow-up was comparable between both intervention groups. Low-risk participants were recruited from previous studies conducted in our laboratory, community daycare centers, local moms' groups, and through announcements posted on a University website. Research staff called parents to briefly describe the research protocol for the present study and confirmed via parent-report that the child had no history of involvement with CPS. If parents expressed interest, a home visit was completed followed by a visit to the laboratory.

Interventions-Parents in the CPS-referred group were randomly assigned to one of two intervention programs. Both interventions were manualized, delivered in the home by trained parent coaches, and consisted of ten 1-hour sessions.

Attachment and Biobehavioral Catch-up (ABC): The ABC intervention was designed to enhance maternal sensitivity to children's distress, increase mother-child synchrony by having mothers follow their children's lead, and decrease frightening behavior. Each session was organized around a particular topic aimed at one of these three components. These components were targeted by parent coaches in several common ways, including presenting a rationale for each component based on research evidence, helping mothers practice the skills during structured activities, commenting on times when the parents provided nurturance to their children's distress or followed their child's lead with delight, and using video feedback to further reinforce target behaviors. Parents received two booster sessions prior to participating in the current study, which were designed to review the three main $\mathrm{ABC}$ targets and refresh the parents' attention to and use of skills.

Developmental Education for Families (DEF): The Developmental Education for Families intervention, borrowed in part from a home visitation program developed by Ramey and colleagues (Ramey, McGuinnes, Cross, Collier, \& Barrie-Blackley, 1982; Ramey, Yeates, \& Short, 1984), was designed to help parents learn ways to enhance children's cognitive and language development. Parents were informed about ways to help their children reach developmental milestones and practiced using themed, developmentally appropriate activities. Parent coaches used video feedback to review skills and demonstrate what the children were gaining from activities. Similar to ABC, DEF parents received two booster sessions prior to participating in the current study. 
Home and lab visits - At a home visit, parents and children were videotaped while they played for 20 minutes using developmentally-appropriate toys from three boxes in a standardized order (NICHD Early Child Care Research Network, 1999, 2003; Vandell, 1979). The interaction task was videotaped for coding of maternal sensitivity. At a lab visit, parents participated in the ERP task. Upon entering the laboratory, research staff briefly explained the procedure, positioned the electrode cap on the mother's head, and prepared electrode sites using conductive paste to minimize impedance.

\section{Measures}

N170 and LPP responses-ERP data were recorded from a 32-channel $\mathrm{Ag} / \mathrm{AgCl}$ electrode cap with placement of electrodes following the International 10-20 System (Cooper, Osselton, \& Shaw, 1969; Jasper, 1958). Continuous EEG was recorded using Advanced Neuro Technology Acquisition Hardware (ANT; Enschede, The Netherlands) and digitized at a rate of 512 samples per second. The task was programmed and administered using Presentation software (Neurobehavioral Systems Inc).

Procedures for the emotional faces ERP task followed those used by Rodrigo et al. (2011). Stimuli included three types of emotional expressions: crying, laughing, and neutral. For each emotion type, 24 images of children's faces under 3 years old were selected, all facing forward and equally distributed across genders (half male, half female) and races (half White, half African American). Following selection criteria of previous studies (Rodrigo et al., 2011), crying faces were characterized by closed or narrowed eyes, furrowed brows, and open mouths with corners lowered; laughing faces were characterized by open eyes, separated and raised brows, and open mouths with corners raised; neutral faces were characterized by open eyes, slight raised brows, and relaxed mouth with closed or halfopened lips. Stimuli were modified to show a close-up of the child's face, insert a black background, remove color, and standardize the image size.

While recording EEG activity, mothers categorized pictures as crying, laughing, or neutral, by pressing a button on a response box. Neutral pictures were always assigned to the center button, whereas crying and laughing pictures were assigned to the right or left button (with button order counterbalanced across participants). Following 12 practice trials, the final set of 72 stimuli (24 crying, 24 laughing, 24 neutral) was presented twice in a random order. Stimuli were displayed for $2000 \mathrm{~ms}$ in the center of the computer screen with an intertrial interval varying between 2000 and $3000 \mathrm{~ms}$.

ERP data were processed using Advanced Source Analysis (ASA) software. The EEG was bandpass filtered between .1 and $30 \mathrm{~Hz}$. Artifact correction was performed for eye blinks and EEG data with peak-to-peak amplitude exceeding $\pm 75 \mu \mathrm{V}$ were rejected. For the LPP, EEG data were re-referenced to the averaged mastoid, whereas an average-reference transformation was used for the N170 (given its proximity to mastoid electrodes). For each mother, average waveforms were calculated from $200 \mathrm{~ms}$ before to $1000 \mathrm{~ms}$ after the stimulus presentation for each stimulus type. The target ERP components (i.e., N170 and LPP) for each mother were measured with reference to her average baseline voltage during the $200 \mathrm{~ms}$ prior to stimulus presentation, by first subtracting the average baseline activity from the entire average. The $\mathrm{N} 170$ was measured as the average amplitude within a time 
window of 140 to $180 \mathrm{~ms}$ at electrodes P7 and P8 (Eimer, Holmes, \& McGlone, 2003;

Rodrigo et al., 2011). The LPP was measured as the average amplitude at the Pz electrode from 300 to 650 ms post-stimulus (Bick, Dozier, Bernard, Grasso, \& Simons, 2013; Schupp et al., 2004).

Maternal sensitivity-The structured play task was coded for maternal sensitivity. Ratings were made on a scale of 1 to 5 using the Qualitative Scales of the Observational Ratings of the Caregiving Environment (ORCE; NICHD Early Child Care Research Network, 1999, 2003). Mothers who received high scores for sensitivity allowed their children to guide the interaction, responded reciprocally and flexibly to their children's behaviors, and attended to the child's signals for engagement and disengagement. In contrast, mothers who received low scores for sensitivity may have dominated the interaction by taking control, teaching the child, and setting the pace, or may have appeared withdrawn or disinterested. Scores for sensitivity ranged from 1.0 to $5.0(M=3.06, S D=$ 1.03 ), and had good inter-rater reliability (ICC $=.81$, for 21 [30\%] double-coded videos). For 35 of the 40 CPS-referred mothers, maternal sensitivity data were also available from videotaped play interactions collected at pre-intervention; as reported below, we included these data to check for baseline equivalence between the intervention groups. Three additional ORCE scales were measured (i.e., positive regard, detachment, and intrusiveness), but these were not used in analyses as our hypotheses were specific to the construct of sensitivity.

Risk factors-Socio-demographic risk data were collected using a questionnaire. Maternal depression was assessed using the depression subscale (6 items) of the Brief Symptoms Inventory (BSI; Derogatis \& Spencer, 1982). The subscale demonstrated good internal consistency $(\alpha=.91)$. Following recommendations from previous studies (e.g., Appleyard, Egeland, van Dulmen, \& Sroufe, 2005), a cumulative risk score was created by totaling the number of socioeconomic risk indicators. Specifically, the following risk indicators were coded dichotomously and totaled to form a composite risk score ranging from 0 to 6 : low income (income-to-needs ratio < 1), ethnic minority group, single parenthood, adolescent mother (at the time of child's birth), low education (less than high school), and elevated maternal depression (t-score > 65). Composite risk scores ranged from 0 to $5(M=2.60, S D$ $=1.48)$. Descriptive statistics for individual risk factors and composite risk scores are presented in Table 2.

\section{Results}

\section{Preliminary Analyses}

Group differences on risk variables-There were significant differences between groups with respect to the composite risk measure, $F(2,67)=16.89, p<.001$. Compared to mothers in the low-risk group, mothers in the CPS-referred ABC group had 1.59 more risk factors on average. Similarly, mothers in the CPS-referred DEF group had 1.91 more risk factors than mothers in the low-risk group. Importantly, mothers in the $\mathrm{ABC}$ and $\mathrm{DEF}$ groups did not differ significantly in their mean number of risk factors, $t(38)=0.86, p>.05$. 
Group differences on maternal sensitivity-At pre-intervention, there were no differences in observed maternal sensitivity between mothers randomly assigned to receive $\mathrm{ABC}$ and those randomly assigned to receive $\mathrm{DEF}, t(33)=-.02, p=.98$. At the time of the present study (post-intervention follow-up), there were significant group differences in maternal sensitivity, $F(2,69)=10.08, p<.001$. Pairwise comparisons showed that DEF mothers scored significantly lower than both $\mathrm{ABC}$ mothers, $t(38)=-3.93, p<.001$, and low-risk mothers, $t(49)=-4.14, p<.001$. Low-risk mothers and ABC mothers did not different in their sensitivity, $t(47)=.25, p=.80$. See Figure 1 .

Preliminary ERP data-Prior to analyses, the quality of ERP data for each participant in each task was examined. Participants were excluded if they had fewer than $50 \%$ useable trials in any of the conditions (due to excessive artifacts) or demonstrated less than $70 \%$ accuracy on the task. Six DEF mothers, 5 ABC mothers, and 4 low-risk mothers were excluded due to less than $50 \%$ useable trials in at least one of the emotion conditions $(<24$ trials); $1 \mathrm{ABC}$ mother was excluded due to scoring less than $70 \%$ accuracy on the task. Thus, final analyses included 70 participants (21 DEF, 19 ABC, 30 Low-risk). We found no differences between mothers with useable ERP data versus mothers with non-useable ERP data. Of a potential of 48 trials for each emotional face condition, participants had a mean of $41.3(S D=7.91), 40.7(S D=7.27)$, and $41.1(S D=7.47)$ neutral, crying, and laughing trials included in analyses. Descriptive statistics of ERP responses are presented in Table 3.

\section{Intervention Effects on ERP Responses}

Group differences in N170 responses during the emotional faces task were examined using a 3 (Group: low-risk, ABC, DEF) x 2 (Electrode: P7, P8) x 3 (Emotion type: crying, laughing, neutral) mixed model ANOVA. There was a main effect of emotion type, $F(2,134)=19.95$, $p<.001$. However, this emotion modulation of the N170 differed by group as shown by a significant 3-way emotion $\mathrm{x}$ electrode $\mathrm{x}$ group interaction, $F(4,134)=4.03, p<.01$ (See Figure 2). In order to probe the nature of this interaction, we conducted separate repeated measures ANOVAs for each group, and examined pairwise comparisons between emotional face conditions. Both low-risk mothers' and ABC mothers' N170 amplitudes at P7 and P8 were modulated by emotion type to a larger degree than DEF mothers. More specifically, the amplitude of low-risk mothers' N170 responses at P7 was significantly modulated by emotion type, $F(2,58)=10.18, p<.001$, with crying faces eliciting a greater negativity than both laughing and neutral faces. For low-risk mothers, emotion differences in N170 amplitude at P8 approached significance, $F(2,58)=2.92, p=.06$, with laughing faces eliciting a greater negativity than both crying and neutral faces. For ABC mothers, N170 amplitude at P7 was significantly modulated by emotion type, $F(2,36)=10.03, p<.001$, with crying and laughing faces eliciting a greater negativity than neutral faces. For $\mathrm{ABC}$ mothers, $\mathrm{N} 170$ amplitude at $\mathrm{P} 8$ was also significantly modulated by emotion type, $F(2,36)=$ $10.94, p<.001$, with crying faces eliciting a greater negativity than both laughing and neutral faces. For DEF mothers, N170 amplitude at P7 was not significantly modulated by emotion type, $F(2,40)=1.96, p>.05$; similarly, N170 amplitude at P8 was not significantly modulated by emotion type, $F(2,40)=0.33, p>.05$. In order to interpret the magnitude of the intervention effect on modulation of the N170, we computed the partial eta squared $\left(\eta^{2} \mathrm{P}\right)$ for the interaction term of intervention group by emotion type (crying versus neutral; 
laughing versus neutral), separately for each electrode. Following Cohen's (1988) conventions for classifying small $\left(\eta_{\mathrm{P}}^{2}=0.02\right)$, medium $\left(\eta_{\mathrm{P}}^{2}=0.13\right)$, and large $\left(\eta_{\mathrm{P}}^{2}=0.26\right)$ effect sizes, this comparison between $\mathrm{ABC}$ and DEF groups in modulation of the N170 by emotion type yielded effect sizes that ranged from small to medium. The effect size of the intervention group by emotion type interaction at $\mathrm{P} 8$ was medium for neutral versus crying faces $\left(\eta_{\mathrm{P}}^{2}=0.18\right)$ and small for neutral versus laughing faces $\left(\eta_{\mathrm{P}}^{2}=0.04\right)$. At P7, the effect size of the intervention group by emotion type interaction was medium for neutral versus laughing faces $\left(\eta_{\mathrm{P}}^{2}=0.11\right)$ and small for neutral versus crying faces $\left(\eta_{\mathrm{P}}^{2}=0.02\right)$.

Group differences in LPP responses during the emotional faces task were examined in a similar way by using a 3 (Group) x 3 (Emotion type) mixed model ANOVA. There was a main effect of emotion type, $F(2,134)=13.04, p<.001$. However, this emotion modulation of the LPP differed by group as shown by a significant emotion $\mathrm{x}$ group interaction, $F(4,136)=2.74, p<.05$ (See Figure 3). In probing this interaction, we found that both lowrisk mothers' and ABC mothers' LPP amplitudes were modulated by emotion type to a larger degree than DEF mothers. Specifically, among low-risk mothers, LPP amplitude was significantly modulated by emotion type, $F(2,58)=8.25, p<.01$, with crying faces eliciting a greater negativity than both laughing and neutral faces. For ABC mothers, LPP amplitude was significantly modulated by emotion type, $F(2,36)=7.42, p<.01$, with crying and laughing faces eliciting a greater positivity than neutral faces. For DEF mothers, LPP amplitude was not significantly modulated by emotion type, $F(2,40)=1.76, p>.05$. The effect sizes of intervention group differences in modulation of the LPP, as calculated following procedures described above, were medium $\left(\eta^{2} P=0.16\right.$ for neutral versus crying, and 0.19 for neutral versus laughing).

\section{Associations Between ERP Responses and Maternal Behavior}

Finally, we examined associations between ERP responses and observed maternal sensitivity. We calculated two difference scores for ERP components of interest: an N170 emotion difference score (i.e., subtracting average N170 to crying and laughing faces from the N170 to neutral faces) and an LPP emotion difference score (i.e., subtracting average LPP to neutral faces from the LPP to crying and laughing faces). Each difference score represented the degree to which emotional faces elicited a larger ERP response than neutral faces. There was a significant positive correlation between maternal sensitivity and the N170 difference score ( $r=.26, p=.03)$, such that as maternal sensitivity increased, the N170 emotion difference score became more positive (i.e., larger N170 to emotional faces relative to neutral faces). The magnitude of this association held when controlling for the composite risk score $(r=.29, p=.02)$. Maternal sensitivity was not significantly associated with the LPP emotion difference score, but was in the expected direction $(r=.22, p=.07)$.

\section{Discussion}

The present study showed that CPS-referred mothers who received the ABC intervention showed enhanced psychophysiological processing of emotional infant faces relative to mothers who received a control intervention. Specifically, ABC mothers' N170 and LPP responses were more strongly modulated by emotion type than DEF mothers' responses, 
partially resembling those of low-risk comparison mothers. Further, maternal sensitivity was correlated with ERP responses, such that more sensitive mothers showed larger ERP responses to emotional faces than neutral faces, compared to less sensitive mothers.

Among typical mothers, children's emotional faces appear to preferentially engage attentional resources. Mothers' processing of children's emotional faces has been suggested to reflect the adaptive value of attending to such cues for promoting infant survival (Rodrigo et al., 2011; Rutherford \& Mayes, 2013; Swain, 2010). The apparent disruption of these attentional processes in CPS-referred mothers may represent a biomarker of the deficits associated with neglectful parenting. Taken together, our findings suggest that CPS-referred mothers may demonstrate specific deficits in physiological processing and behavioral responses to children's emotional cues, and these deficits appear amenable to change following a parenting intervention.

Notably, mothers who received the $\mathrm{ABC}$ intervention demonstrated enhanced attention to both crying and laughing expressions. The enhanced processing of laughing expressions was relatively unique to this group, in that low-risk mothers in the Rodrigo et al. (2011) study as well as low-risk mothers in the present study did not consistently demonstrate larger ERP responses to laughing faces than neutral faces. The $\mathrm{ABC}$ intervention aims to increase maternal responsiveness to children's cues of distress as well as children's signals of positive affect and social engagement. Thus, by helping mothers engage in synchronous interactions, it is possible that the $\mathrm{ABC}$ intervention influences how mothers process both positive and negative dimensions of children's emotion expression.

In previous studies, the $\mathrm{ABC}$ intervention has been shown to be effective in enhancing attachment quality and cortisol regulation among children living with their CPS-referred birth parents (Bernard et al., 2012; Bernard et al., in press), with medium effect sizes found for these outcomes. The present study suggests that changes may also occur in CPS-referred mothers' psychophysiological responsiveness to child cues as a result of the $\mathrm{ABC}$ intervention. The effect sizes for the present study ranged from small to medium, with their magnitude varying by emotion type (i.e., crying or laughing), ERP component (i.e., N170 or LPP), and scalp location (i.e., left vs. right, for the N170). Whereas the ABC intervention demonstrated a consistently medium effect size on enhancing the LPP to both crying and laughing faces, the effect size for the N170 was more variable. Specifically, the ABC intervention had a medium effect on the N170 to crying faces when measured in the right hemisphere (P8), but a small effect on the N170 to crying faces when measured in the left hemisphere (P7). Further, the ABC intervention had a small effect on the N170 to laughing faces when measured in the right hemisphere (P8), but a medium effect on the N170 to laughing faces when measured in the left hemisphere (P7). It is possible that these differences in effect sizes reflect true hemispheric differences in how the $\mathrm{ABC}$ intervention influences mothers' processing of children's cues; however, replication of these effects in addition to further examination of maternal brain responses more generally, would be needed to further examine this possibility. Additionally, it is consider that there is mixed evidence in the broader literature about the sensitivity of the N170 to differentiating between emotional expressions. Although a number of studies have found larger N170 responses to emotional faces than neutral faces in typical adult samples (e.g., Batty \& Taylor, 2003; Eger, 
Jedyak, Iwaki, \& Skrandies, 2003; Pizzagalli, Lehmann, Koening, Regard, \& PascualMargui, 2000), other studies have found no N170 differences by type of facial expression (e.g., Dennis, Malone, \& Chen, 2009; Recio, Sommer, \& Schact, 2011). Thus, it is possible that the small effect sizes observed in certain comparisons in the present study reflect modest sensitivity of the N170 to different emotional expressions in general. The differences in ERP responses between ABC and DEF mothers were apparent several years after the completion of the intervention (with booster sessions), as data collection for the present study occurred between 1.4 and 5.2 years following the 10-session intervention ( $M=3.61$ years, $S D=0.73$ ). Given the timing of assessments in the current study, we cannot determine when the differences in ERP responses between ABC and DEF groups emerged. Measuring mothers' ERP responses at multiple time-points (e.g., pre-intervention, immediately post, 1 year-follow-up, and long-term follow-up) could further clarify when changes occur and their persistence over time. Future studies can also examine whether changes in ERP responses mediate pathways towards improved child outcomes.

The present study benefitted from a number of methodological strengths, including random assignment to treatment group and the inclusion of a low-risk comparison group. Despite these strengths, certain limitations should be considered when interpreting the results of this study. First, our sample size was relatively small, especially for testing 3-way interactions. Second, we did not have pre-intervention measures of ERP activity to confirm baseline equivalence or examine within-individual change. Future studies could benefit from measurement of ERP responses across multiple assessments, including pre- and postintervention. Third, although the randomized control design assumes that groups did not differ on key variables prior to participating in the intervention, average income was higher among mothers in the $\mathrm{ABC}$ intervention than DEF mothers. $\mathrm{ABC}$ and DEF groups were comparable on all risk indicators and baseline maternal sensitivity. Finally, mothers varied widely with regard to parenting history, such as number of biological children (ranging from 1 to 13), how long they had been a mother, and time since their most recent child was born (with some currently caring for an infant). Previous studies examining the neurobiology of maternal behavior have often applied stringent criteria for the target sample, such as only including first-time mothers or defining a specific period of motherhood (e.g., initial postnatal period). On one hand, the heterogeneity with regard to mothering experiences of participants makes it challenging to place our findings in the context of previous studies. On the other hand, our compelling findings despite a noisy sample suggest that the differences may be robust and generalizable across mothers with a range of previous parenting experiences.

In conclusion, findings from the current study demonstrate that a short-term attachmentbased parenting intervention enhanced mothers' psychophysiological processing of emotional faces. Our findings add to a growing body of research about the neurobiology of the maternal care by helping us better understand problematic parenting.

\section{Acknowledgments}

The project described was supported by funding to M. Dozier from the National Institute of Mental Health (Award numbers: R01MH052135, R01MH074374, and R01MH084135) and funding from Edna Bennett Pierce. 


\section{References}

Appleyard K, Egeland B, van Dulmen MHM, Sroufe LA. When more is not better: The role of cumulative risk in child behavior outcomes. Journal of Child Psychology and Psychiatry. 2005; 46:235-245.10.1111/j.1469-7610.2004.00351.x [PubMed: 15755300]

Barrett J, Fleming AS. Annual research review: All mothers are not created equal: Neural and psychobiological perspectives on mothering and the importance of individual differences. Journal of Child Psychology and Psychiatry. 2011; 52:368-397.10.1111/j.1469-7610.2010.02306.x [PubMed: 20925656]

Batty M, Taylor MJ. Early processing of the six basic facial emotional expressions. Cognitive Brain Research. 2003; 17:613-620.10.1016/S0926-6410(03)00174-5 [PubMed: 14561449]

Bernard K, Dozier M, Bick J, Gordon MK. Normalizing blunted diurnal cortisol rhythms among children at risk for neglect: The effects of an early intervention. Development and Psychopathology. In press.

Bernard K, Dozier M, Bick J, Lewis-Morrarty E, Lindhiem O, Carlson E. Enhancing attachment organization among maltreated children: Results of a randomized clinical trial. Child Development. 2012; 83:623-636.10.1111/j.1467-8624.2011.01712.x [PubMed: 22239483]

Bick J, Dozier M, Bernard K, Grasso D, Simons. Foster mother-infant bonding: Associations between foster mothers' oxytocin production, electrophysiological brain activity, feelings of commitment, and caregiving quality. Child Development. 2013; 84:826-840. doi:10.1111.cdev.12008. [PubMed: 23163703]

Cicchetti D, Rogosch FA, Toth SL. Fostering secure attachments in infants in maltreating families through preventive interventions. Development and Psychopathology. 2006; 18:623-649.10.1017/ S0954579406060329 [PubMed: 17152394]

Cohen, J. Statistical power analysis for the behavioral sciences. 2. Hillsdale, NJ: Erlbaum; 1988.

Dennis TA, Malone MM, Chen CC. Emotional face processing and emotion regulation in children: An ERP study. Developmental Neuropsychology. 2009; 34:85-102.10.1080/87565640802564887 [PubMed: 19142768]

Derogatis, LR.; Spencer, PM. Administration and Procedures: BSI Manual I. Baltimore, MD: Clinical Psychometrics Research; 1982.

De Wolff M, van IJzendoorn MH. Sensitivity and attachment: A meta-analysis on parental antecedents of infant attachment. Child Development. 1997; 68:571-591.10.2307/1132107 [PubMed: 9306636]

Dozier, M. the Infant Caregiver Project. Unpublished manuscript. University of Delaware, Department of Psychology; Newark, DE: 2006. Attachment and Biobehavioral Catch-up Intervention.

Edwards A, Shipman K, Brown A. The socialization of emotion understanding: A comparison of neglectful and nonneglectful mothers and their children. Child Maltreatment. 2005; 10:293304.10.1177/1077559505278452 [PubMed: 15983112]

Eger E, Jedyak A, Iwaki T, Skrandies W. Rapid extraction of emotional expression: Evidence from evoked potential fields during brief presentation of face stimuli. Neuropsychologia. 2003; 41:808817.10.1016/S0028-3932(02)00287-7 [PubMed: 12631531]

Eimer M, Holmes A, McGlone FP. The role of spatial attention in the processing of facial expression: An ERP study of rapid brain responses to six basic emotions. Cognitive, Affective, \& Behavioral Neuroscience. 2003; 3:97-110.10.3758/CABN.3.2.97

Feingold A. Effect sizes for growth-modeling analysis for controlled clinical trials in the same metric as for classical analysis. Psychological Methods. 2009; 14:43-53.10.1037/a0014699 [PubMed: 19271847]

Gaudin JM, Polansky NA, Kilpatrick AC, Shilton P. Family functioning in neglectful families. Child Abuse \& Neglect. 1996; 20:363-377.10.1016/0145-2134(96)00005-1 [PubMed: 8730772]

Hajcak G, MacNamara A, Olvet DM. Event-related potentials, emotion, and emotion regulation: An integrative review. Developmental Neuropsychology. 2010; 35:129155.10.1080/87565640903526504 [PubMed: 20390599] 
Hesse E, Main M. Frightened, threatening, and dissociative parental behavior in low-risk samples: Description, discussion, and interpretations. Development and Psychopathology. 2006; 18:309343.10.1017/S0954579406060172 [PubMed: 16600057]

Hussey J, Marshal J, English D, Knight E, Lau A, Dubowitz H, Kotch JB. Defining maltreatment according to substantiation: Distinction without a difference? Child Abuse and Neglect. 2005; 29:479-492.10.1177/1077559504269192 [PubMed: 15970321]

Karna A, Voeten M, Little TD, Poskiparta E, Kalijonen A, Salmivalli C. A large-scale evaluation of the KiVa Antibullying Program: Grades 4 - 6. Child Development. 2011; 82:311-330.10.1111/j. 1467-8624.2010.01557.x [PubMed: 21291444]

Lind T, Bernard K, Ross E, Dozier M. Intervention effects on negative affect of CPS-referred children: Results of a randomized clinical trial. Child Abuse \& Neglect. 2014 epub. 10.1016/j.chiabu. 2014.04.004

Lorberbaum JP, Newman JD, Horwitz AR, Dubno JR, Lydiard RB, Hamner MB, George MS. A potential role for thalamocingulate circuitry in human maternal behavior. Biological Psychiatry. 2002; 51:431-445.10.1016/S0006-3223(01)01284-7 [PubMed: 11922877]

Lyons-Ruth K, Connell DB, Zoll D, Stahl J. Infants at social risk: Relations among infant maltreatment, maternal behavior, and infant attachment behavior. Developmental Psychology. 1987; 23:223-232.10.1037/0012-1649.23.2.223

Milot T, St-Laurent D, Eithier LS, Provost MA. Trauma-related symptoms in neglected preschoolers and affective quality of mother-child communication. Child Maltreatment. 2010; 15:293304.10.1177/1077559510379153 [PubMed: 20930179]

NICHD Early Child Care Research Network. Child care and mother-child interactions in the first 3 years of life. Developmental Psychology. 1999; 35:1399-1413.10.1037/0012-1649.35.6.1399 [PubMed: 10563730]

NICHD Early Child Care Research Network. Early child care and mother-child interaction from 36 months through first grade. Infant Behavior and Development. 2003; 26:345-370.10.1016/ S0163-6383(03)00035-3

Noriuchi M, Kikuchi Y, Senoo A. The functional neuroanatomy of maternal love: Mother's response to infant's attachment behaviors. Biological Psychiatry. 2008; 63:415-423.10.1016/j.biopsych. 2007.05.018 [PubMed: 17686467]

Pizzagalli D, Lehmann D, Koenig T, Regard M, Pascual-Marqui RD. Face-elicited ERPs and affective attitude: Brain electric microstate and tomography analyses. Clinical Neurophysiology. 2000; 111:521-531.10.1016/S1388-2457(99)00252-7 [PubMed: 10699416]

Proverbio AM, Brignone V, Matarazzo S, Del Zotto M, Zani A. Gender and parental status affect the visual cortical response to infant facial expression. Neuropsychologia. 2006; 44:29872999.10.1016/j.neuropsychologia.2006.06.015 [PubMed: 16879841]

Ramey, CT.; McGinness, GD.; Cross, L.; Collier, AM.; Barrie-Blackley, S. The Abecedarian approach to social competence: Cognitive and linguistic intervention for disadvantaged preschoolers. In: Borman, K., editor. The social life of children in a changing society. Hillsdale, NJ: Erlbaum; 1982. p. 14-173.

Ramey CT, Yeates KO, Short EJ. The plasticity of intellectual development: Insights from preventative intervention. Child Development. 1984; 55:1913-1925.10.2307/1129938 [PubMed: 6510061]

Recio G, Sommer W, Schact A. Electrophysiological correlates of perceiving and evaluating static and dynamic facial emotional expressions. Brain Research. 2011; 1376:66-75.10.1016/j.brainres. 2010.12.041 [PubMed: 21172314]

Rodrigo MJ, Leon I, Quinones I, Lage A, Byrne S, Bobes MA. Brain and personality bases of insensitivity to infant cues in neglectful mothers: An event-related potential study. Development and Psychopathology. 2011; 23:163-176.10.1017/S0954579410000714 [PubMed: 21262046]

Rutherford, HJ.; Mayes, L. Maternal perception and regulation of infant affect: Evidence from electroencephalography. Paper presented at the biennial meeting of the Society for Research in Child Development; Seattle, WA. Apr. 2013 
Schupp HT, Cuthbert BN, Bradley MM, Hillman CH, Hamm AO, Lang PJ. Brain processes in emotional perception: Motivated attention. Cognition and Emotion. 2004; 18:593611.10.1080/02699930341000239

Swain JE. The human parental brain: In vivo neuroimaging. Progress in Neuro-Psychopharmacology \& biological Psychiatry. 201010.1016/j.pnpbp.2010.10.017

Swain JE, Lorberbaum JP, Kose S, Strathearn L. Brain basis of early parent-infant interactions: Psychology, physiology, and in vivo functional neuroimaging studies. Journal of Child Psychology and Psychiatry. 2007; 48:262-287.10.1111/j.1469-7610.2007.01731.x [PubMed: 17355399]

Vandell DL. Effects of a playgroup experience on mother-son and father-son interaction. (1979). Developmental Psychology. 1979; 15:379-385.10.1037/0012-1649.15.4.379 


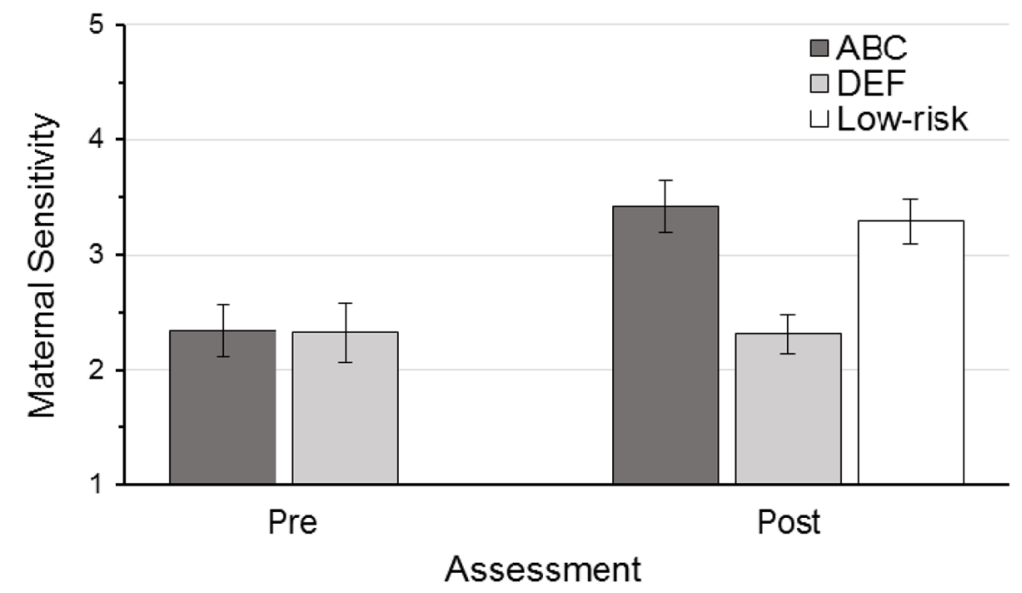

Figure 1.

Group differences in maternal sensitivity at pre-intervention (baseline) and post-intervention follow-up (concurrent with event-related potentials (ERP) assessment) for the Attachment and Biobehavioral Catch-up (ABC; experimental intervention) group, Developmental Education for Families (DEF; control intervention) group, and Low-risk comparison group 
(a) Low-risk

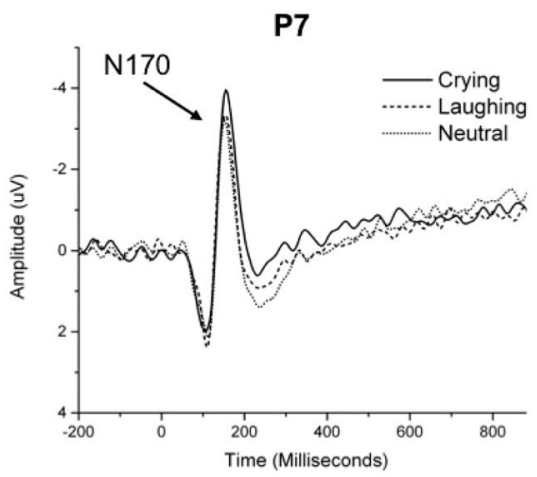

(b) CPS-referred $A B C$

(c) CPS-referred DEF
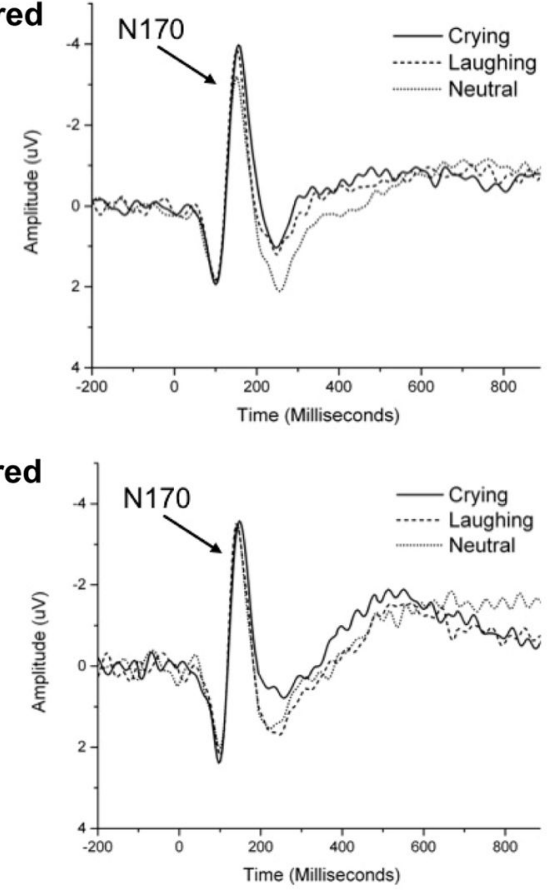

Figure 2.
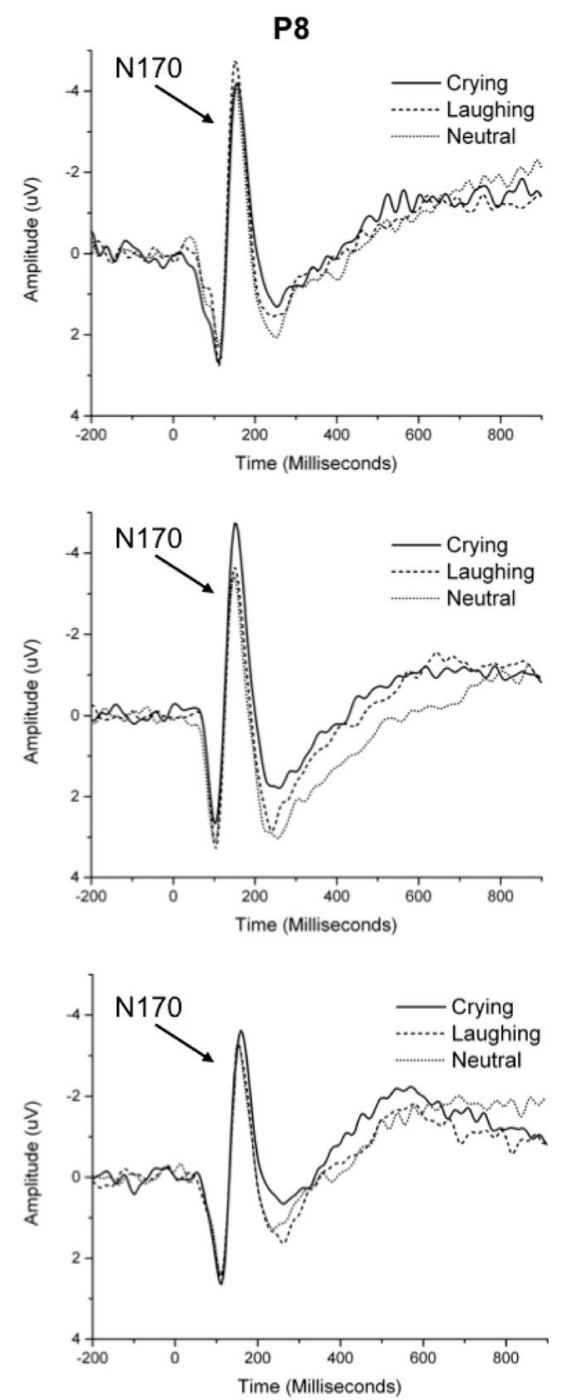

Grand-averaged event-related potentials (ERP) of low-risk, Child Protective Servicesreferred (CPS-referred) ABC (Attachment and Biobehavioral Catch-up), and CPS-referred DEF (Developmental Education for Families) mothers while viewing crying, laughing, and neutral children's faces in the emotional faces task. Left (P7) and right (P8) electrodes were selected for measuring the N170 in the time window from $140-180 \mathrm{~ms}$. 
(a) Low-risk

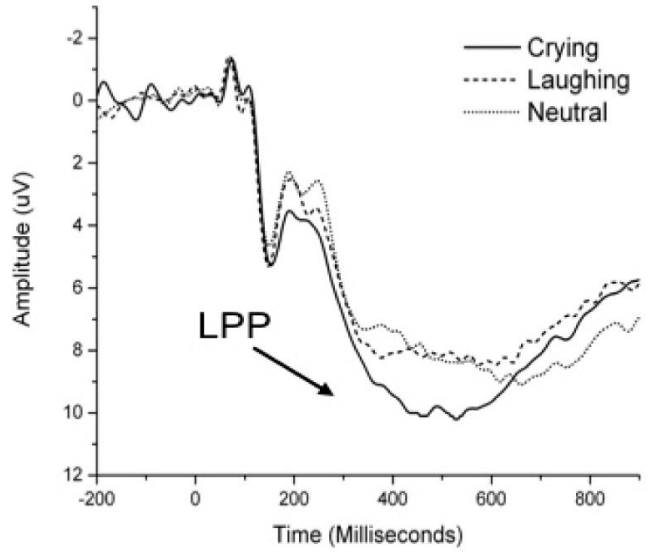

(b) CPS-referred $A B C$

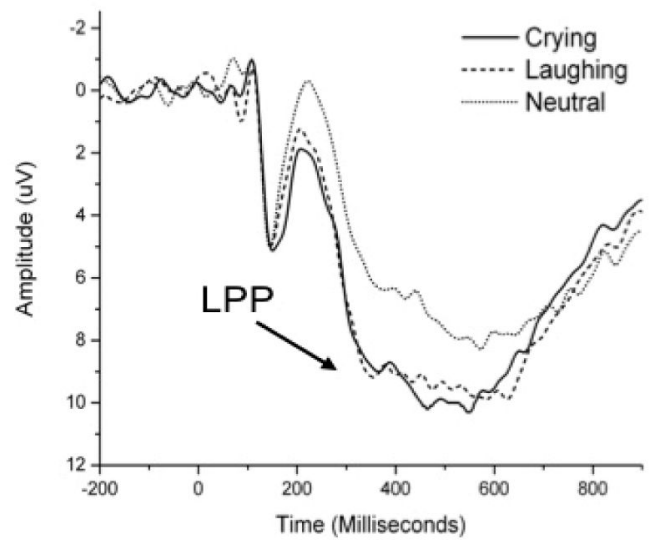

(c) CPS-referred DEF

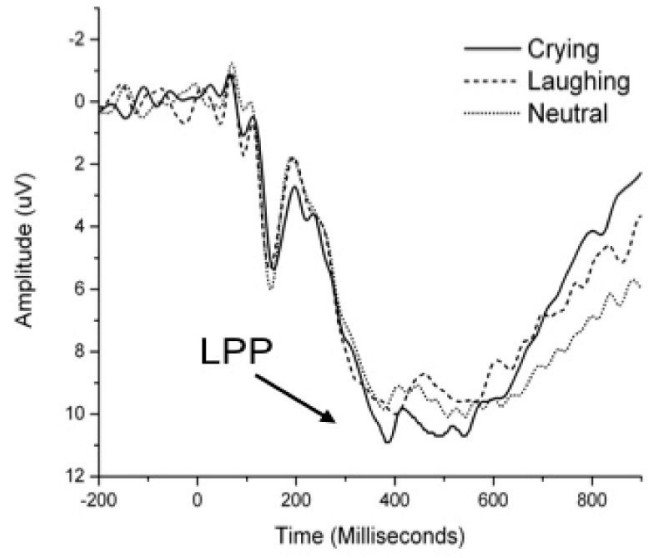

Figure 3.

Grand-averaged event-related potentials (ERP) of low-risk, Child Protective Servicesreferred (CPS-referred) ABC (Attachment and Biobehavioral Catch-up), and CPS-referred DEF (Developmental Education for Families) mothers while viewing crying, laughing, and neutral children's faces in the emotional faces task. The Pz electrode was selected for measuring the late positive potential (LPP) in the time window from $300-650 \mathrm{~ms}$. 


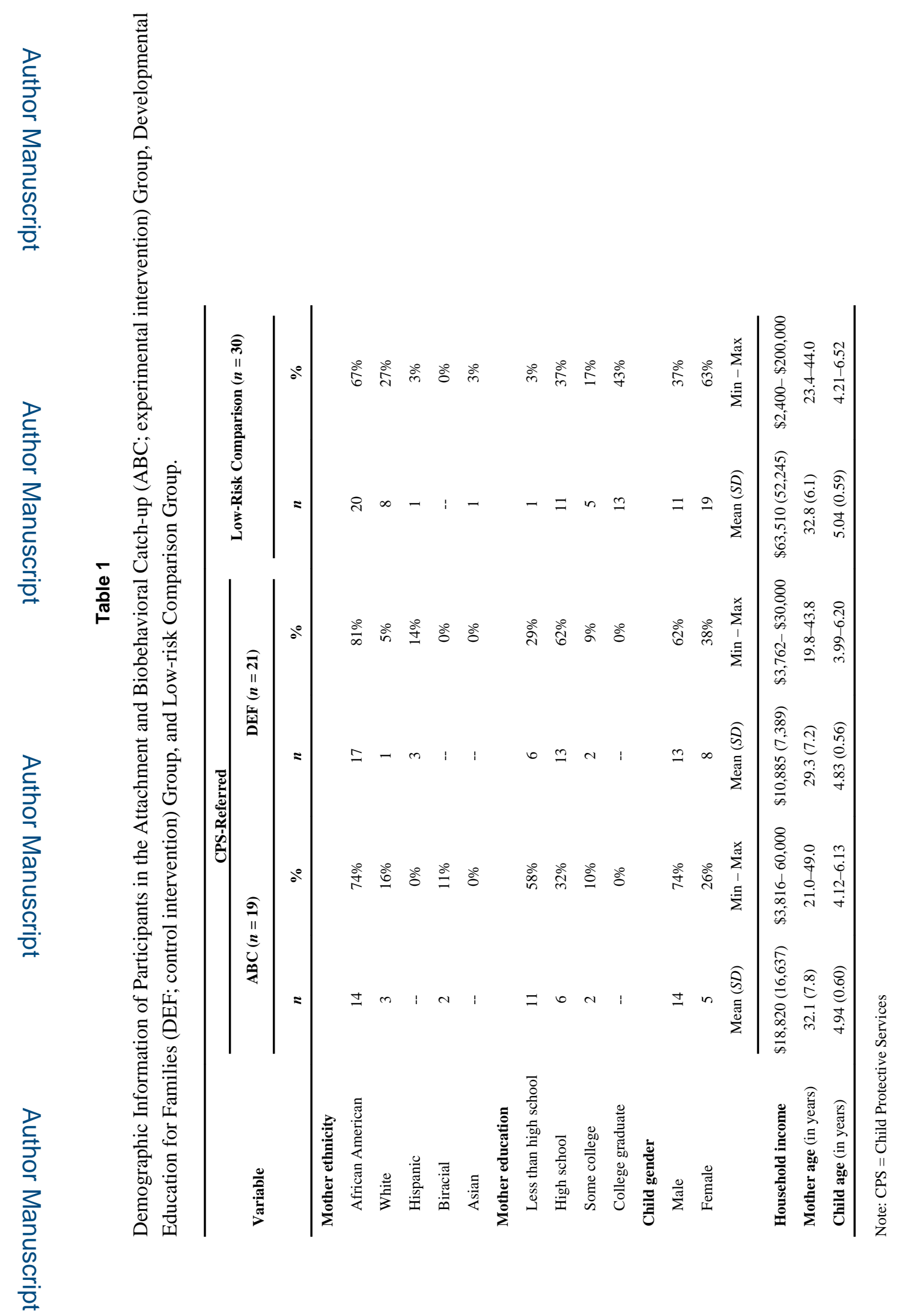

Child Dev. Author manuscript; available in PMC 2016 November 01. 


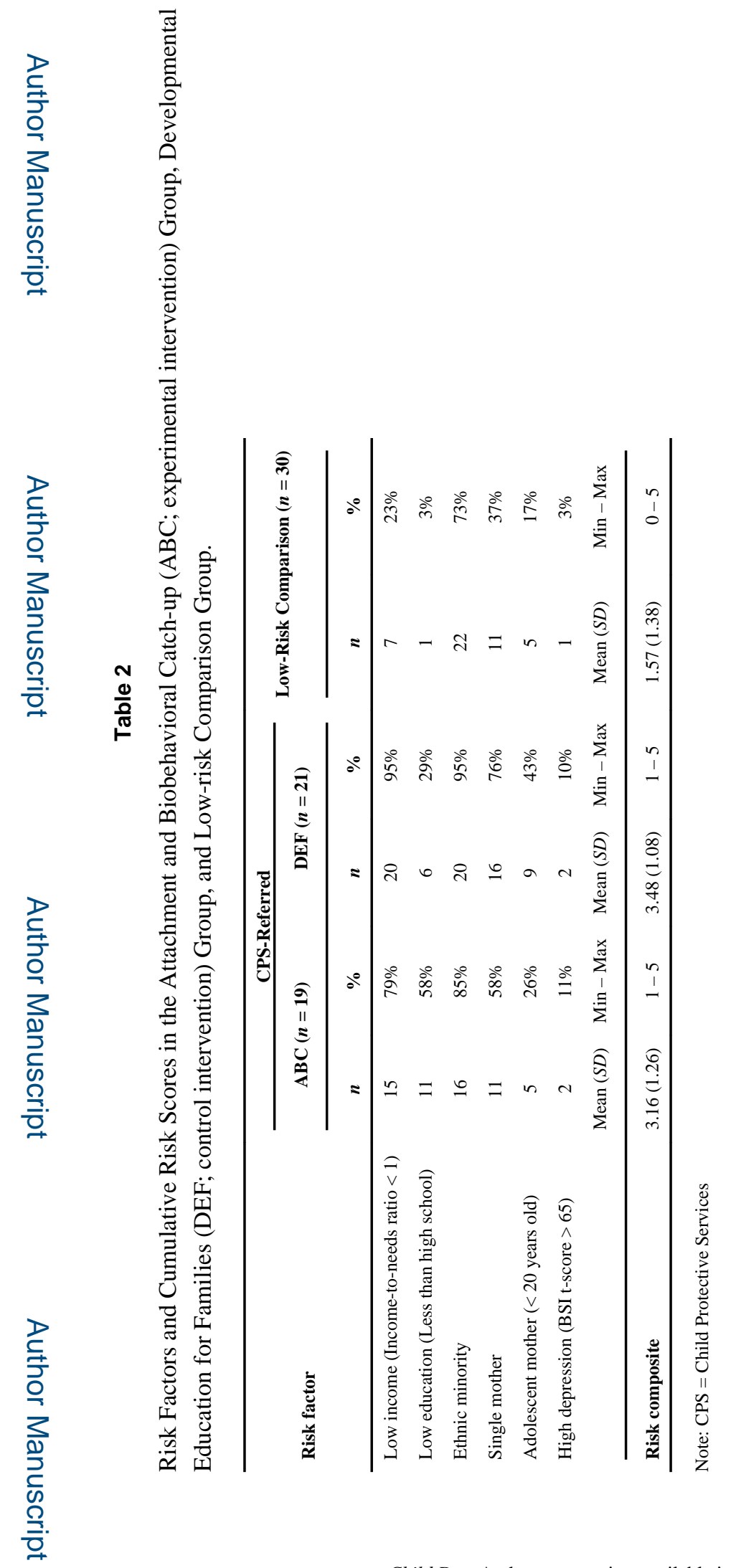

Child Dev. Author manuscript; available in PMC 2016 November 01. 


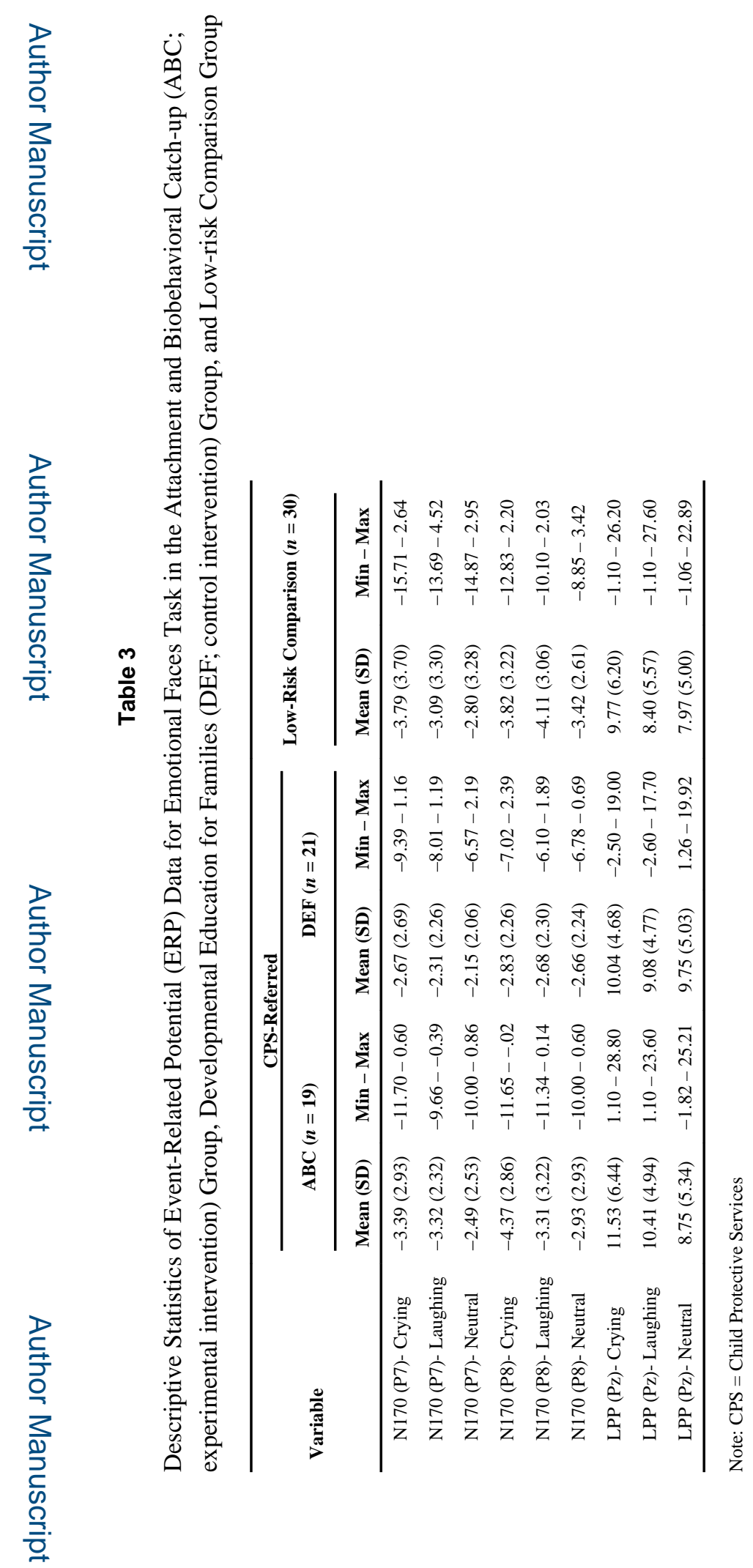

Child Dev. Author manuscript; available in PMC 2016 November 01. 